\title{
FLORE (PTERIDOPHYTA ET SPERMATOPHYTA) DES ZONES HUMIDES DU MAROC MÉDITERRANÉEN: INVENTAIRE ET ECOLOGIE
}

\author{
Abdeslam ENNABILI et Mohammed ATER
}

\begin{abstract}
RESUME. Flore (Pteridophyta et Spermatophyta) des zones humides du Maroc méditerranéen: Inventaire et écologie. Malgré l'intérêt qu'on porte de plus en plus aux zones humides à travers le monde, peu d'études ont été consacrées à la flore hygrophile marocaine. Après avoir prospecté 45 stations, on a évalué la diversité spécifique des hygrophytes du Maroc méditerranéen à partir de 70 relevés floristiques. Sur les 389 espèces reconnues dans cette étude, 175 espèces sont caractéristiques des zones humides. Les espèces rares ou nouvellement citées ont été soulignées. D'autre part, les phytocénoses observées ont été classées en fonction de la physionomie et de la composition spécifique. Une attention particulière a été consacrée à la discussion des principaux facteurs écologiques influant sur les groupements hygrophiles à savoir : la submersion, la salinité, la charge organique domestique, le mésoclimat et les travaux d'aménagement. Ainsi, on a pu regrouper les espèces dominantes en classes écologiques.
\end{abstract}

Mots clés: Flore hygrophile, Pteridophyta, Spermatophyta, zone humide, Maroc méditerranéen, écologie, diversité spécifique, phytocénose.

\begin{abstract}
Wetland flore (Pteridophyta and Spermatophyta) of Mediterranean Morocco. Inventory and ecology. Despite the interest that one wears increasingly to wetlands through the world, few studies have been devoted to Moroccan wetland flora. After having prospected 45 sites, we have evaluated the specific diversity of wetland flora from 70 floristic inventories. Among 389 recognised species in this study, 175 are wetland species. Rare species or newly quoted have been underlined. On the other hand, the observed plant groupings have been classified according to the physiognomy and the specific composition. A particular attention has been devoted to the discussion of the main ecological factors affecting wetland plant groupings namely : the submersion, the salinity, the domestic organic loading, the local climate and the anthropic perturbations. Thereby, we have ranked dominant species in some ecological groups.
\end{abstract}

Key words : wetland flore, Pteridophyta, Spermatophyta, Mediterranean Morocco, ecology, specific diversity.

\section{INTRODUCTION}

Les zones humides ont été définies par la convention de Ramsar (convention relative aux zones humides d'importance internationale, 1971) comme " des étendues de marais, de fagnes, de tourbières ou d'eaux naturelles ou artificielles, permanentes ou temporaires, où l'eau est stagnante ou courante, douce, saumâtre ou salée, y compris des étendues d'eau marine dont la profondeur n'excède pas six mètres". En se basant sur cette définition, 
on peut classer les zones humides du Maroc méditerranéen en:

a) Zones humides naturelles: elles se présentent sous diverses formes à savoir les lacs, les lagunes, les marais, les sebkhas, les sources ponctuelles ou diffuses, les tourbières, les zones de suintement et les cours d'eau;

b) Zones humides artificielles: elles comprennent les retenues des barrages, les points de rejet d'effluents domestiques ou industriels, les zones d'irrigation, etc.

Sous l'impulsion de diverses conventions internationales de protection de la nature (OUA, 1968; Ramsar, 1971; UNESCO, 1972; Washington, 1973; CEE, 1979; Bern, 1979 et Bonn, 1979), les zones humides suscitent de plus en plus l'intérêt de la communauté scientifique internationale. Au Maroc, d'une part, cet intérêt est particulièrement orienté vers l'avifaune, et d'autre part, il concerne surtout la façade atlantique. En effet, quatre zones humides de la façade atlantique ont été inscrites sur la liste de la convention de Ramsar: Merja Zerga (280 ha), Merja Sidi Boughaba (650 ha), lac Afenourir (280 ha) et la lagune Khnifiss (6500 ha). Dans le cas de la façade méditerranéenne, malgré la diversité des types de zones humides et la reconnaissance de cinq sites (embouchure Moulouya, sebkha Bou Areg, oued Tahaddarte, lagune et barrage Smir) comme sites à intérêts biologique et écologique (BCEOM-SECA, 1994), aucun site ne bénéficie actuellement d'un statut de réserve naturelle.

Du point de vue botanique et phytoécologique, exceptés quelques travaux d'ordre général (Emberger \& Maire, 1941; Maire, 1952/1980; De la Torre, 1955, Ionesco \& Stefanesco, 1967, Sauvage, 1971 et Benabid, 1983) ou plus spécialisés (Nègre, 1956; Atbib, 1979 et Allport et al., 1994), la flore hygrophile marocaine demeure très peu étudiée. En effet, cette étude constitue une contribution à la connaissance de la flore des zones humides du Maroc méditerranéen et à l'évaluation de la biodiversité à l'échelle régionale.

\section{MATERIELS ET METHODES}

Le secteur d'étude couvre toute la région Nord du Maroc comprise entre les longitudes $2^{\circ}$ et $6^{\circ} \mathrm{W}$, et les latitudes $35^{\circ}$ et $36^{\circ} \mathrm{N}$ (Fig. 1 ). Du point de vue climatique et floristique, ce secteur présente une grande diversité. En effet, à part le saharien, on y rencontre tous les bioclimats méditerranéens (Benabid, 1982) avec une nette dominance du bioclimat aride dans la partie orientale qui est moins humide que la zone occidentale. L'étage de végétation le plus étendu dans la zone d'étude est le thermoméditerranéen qui est suivi en moindre importance successivement par le mésoméditerranéen, le supraméditerranéen, le montagnard-méditerranéen l'oroméditerranéen (Achhal et al., 1990 et Benabid, 1983, 1984). Concernant les séries de végétation en rapport avec notre sujet d'étude, on peut citer en altitude une série à Prunus lusitanica pour les formations ripicoles des cours d'eau et des sources; alors qu'à des altitudes moins importantes, on cite une série thermoméditerranéenne de Fraxinus angustifolia pour les ripisylves (Benabid, 1983).

Les sols les plus couramment rencontrés sont de type argileux, argilo-sableux à sablonneux ou vaseux. Toutefois, les sites pollués par les eaux usées sont très riches en vase organique. Le substratum qui affleure au niveau des berges des parties en amont des oueds et au niveau des sources est dominé selon les cas par les grès, les pélites, les schistes, les calcaires, les conglomérats tilliteux ou la péridotite.

Des campagnes de prospection ont été organisées pendant les périodes végétatives de 1994 et 1995. Ainsi, 70 relevés floristiques ont été réalisés dans 45 stations considérées comme représentatives du Maroc méditerranéen (fig.1). A l'exception des stations 14 et 36 qui appartiennent respectivement aux mésoméditerranéen et supraméditerranéen, 


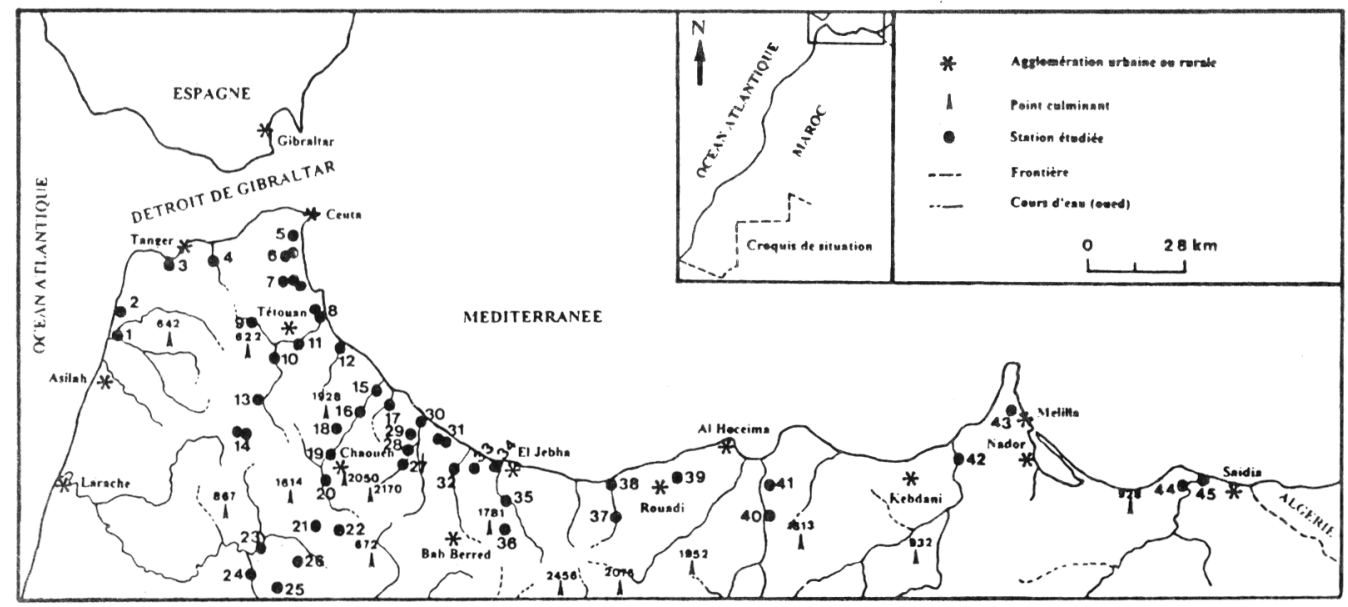

Figure 1. Zone d'étude. Study area.Toponymie des stations étudiées. (o., oued; R., relevé) : Tanger : 1 , embouchure de l'o. Tahaddarte (R.1 à 3); 2, Daya Sghira (R.4); 3, o. Msabene-Sania (R.5 et 6); 4 , o. Lediane; Tétouan: 5, Riffiene; 6, Negro (R.7 à 12), Allyine (R.13), Frassou (R.14); 7, Smir (R.15 à 29) , M'diq (R.30 et 31); 8, o. Malah, o. Martil (R.32); 9, El Fendek; 10, Mhajrat; 11, M'hannech, Bouanane (R.33 à 35); 12, o. Amsa; 13, o. El Kbir; 14, Jbel Alam (R.36 à 38); Chaouen : 15-16, Oued Laou (R.39 et 40); 17, Targa; 18, Outa Tirines (R.41); 19, Bge Talambote; 20, Sifillaow (R.42 à 43), Grankha (R.44), Ain Errami (R.45); 21, Zourak (R.46); 22, Resana; 23, Aaniçar Messous (R.47 et 48); 24, Ltaoua (R.49 et 50); 25, Metmoura (R.51), Outa Messari (R.52 et 53); 26, Chebtal; 27, o. Kannar-Icheritene (R.54); 28, o. Kannar-Sk Had (R.55); 29, Bouhmed; 30, o. Tihissas (R.56 et 58), Stehat (R.59 et 60), Chmaala (R.61); El Jebha : 31, o. Ichendirene (R.62), o. Sidi Aarabene (R.63); 32, o. Mter; 33, Jnane Niche; 34, embouchure de l'o. Ouringa; 35, o. Tazemmourt; 36, Ouzarhar (R.64); Beni Boufrah : 37, Ras Alkala; 38, o. Benii Boufrah; 39, Bakyoua (R.65); Al Hoceima : 40, o. Nekôr-Tamellaht (R.66); 41, Bge M.A. El Khattabi; Kebdani : 42, o. Kert-Hammam (R.67); Nador : 43, Idouhdouhan (R.68); Saïdia : 44, Aïn Beïda; 45, o. Moulouya-Chararba (R.69 et 70). Toponomy of studied sites.

l'ensemble des stations appartiennent à l'étage de végétation thermoméditerranéen.

Pour chaque relevé, on a retenu les principales données relatives à la station, au couvert végétal et aux facteurs anthropozoogènes majeurs (tab. 1). Les indices d'abondance-dominance et de sociabilité ont été établis selon l'école sigmatiste (Braun Blanquet, 1968).

Mises à part quelques exceptions, la nomenclature taxonomique adoptée est celle de la Flora Europaea (Tutin et al. 1990/1993). Pour des cas particuliers et pour la nomenclature infraspécifique, on s'est référé à Maire (1952/1980) et Quézel \& Santa (1962/ 1963).
Chaque relevé a été décrit par la présence ou l'absence des espèces considérées comme dominantes. L'ensemble des données correspond alors à un tableau disjonctif à codage logique. Vu la nature des données, c'est une Analyse Factorielle des Correspondances (AFC) «Relevés-Espèces dominantes » qui constitue l'approche la plus appropriée pour mettre en évidence d'éventuels groupes écologiques. Le logiciel utilisé est STAT-ITCF. D'autre part, l'étude de la corrélation entre le pourcentage des hygrophytes et la submersion a été réalisée en utilisant le logiciel SPSS/PC ${ }^{+}$.

Des échantillons témoins des espèces déterminées sont conservés au Département de 


\begin{tabular}{|c|c|c|c|c|c|c|c|}
\hline $\mathbf{R}$ & $\mathbf{S}$ & $\mathrm{Al}$ & Sb & Aa & $\mathrm{Nt}$ & $\mathrm{Nh}$ & Espèces dominantes (ad.s) \\
\hline 1 & 1 & $<5$ & $0-50 *$ & PÍ & 1 & 100 & Zostera noltii (5.5) \\
\hline 2 & i & $<5$ & $0-25 *$ & $\mathrm{P}$ & 1 & 100 & Spartina maritima $(5.5)$ \\
\hline 3 & 1 & $<5$ & 0 & $\mathrm{P}$ & 11 & 33.3 & Arthrocnemum fruticosum (3.3) \\
\hline 4 & 2 & $<25$ & 0 & $\mathrm{~B}, \mathrm{P}$ & 21 & 19 & Juncus maritimus (4.2) \\
\hline 5 & 3 & $<25$ & $0-6$ & A,P & 36 & 83.3 & Nerium oleander (2.4), Tamarix africana (2.4) \\
\hline 6 & 3 & $<5$ & 11 & E,P & 9 & 55.6 & Typha angustifolia (5.5) \\
\hline 7 & $6 a$ & $<5$ & $>100$ & A & 2 & 100 & Potamogeton pectinatus (5.5) \\
\hline 8 & $6 a$ & $<5$ & $0-60$ & A,P & 13 & 76.9 & Tamarix gallica (3.4). Paspalum vaginatum (3.4) \\
\hline 9 & $6 a$ & $<10$ & 0 & $\mathrm{~A}, \mathrm{P}$ & 17 & 82.4 & Tamarix gallica (3.4), Scirpus maritimus (3.1) \\
\hline 10 & $6 a$ & $<10$ & 16 & $\mathrm{~A}, \mathrm{D}, \mathrm{P}$ & 7 & 100 & Scirpus litoralis (3.1), S. maritimus (3.1) \\
\hline 11 & $6 \mathrm{a}$ & $<5$ & $0-40$ & $\mathrm{~A}, \mathrm{C}$ & 16 & 100 & Juncus maritimus (3.2), Puccinellia sp. (3.3) \\
\hline 12 & $6 a$ & $<5$ & $0->70$ & A,D & 29 & 37.9 & Phragmites australis (3.4), Juncus maritimus (3.2) \\
\hline 13 & $6 b$ & $<10$ & 12 & $A, P$ & 16 & 62.5 & $\begin{array}{l}\text { Juncus articulatus }(3.1), \\
\text { Eryngium corniculatum (3.1) }\end{array}$ \\
\hline 14 & $6 \mathrm{c}$ & $<25$ & $0-15$ & $\mathrm{P}$ & 19 & 78.9 & Iris pseudacorus (5.4) \\
\hline 15 & $7 \mathrm{a}$ & $<5$ & $10-35$ & E,P & 22 & 86.4 & $\begin{array}{l}\text { Scirpus litoralis (2.1), S. maritimus (2.1), } \\
\text { Juncus maritimus (2.2) }\end{array}$ \\
\hline 16 & $7 \mathrm{a}$ & $<5$ & $5-45$ & $\mathrm{Ch}, \mathrm{E}, \mathrm{P}$ & 20 & 80 & Scirpus litoralis (3.4), Lemna gibba (3.4) \\
\hline 17 & $7 \mathrm{a}$ & $<5$ & $0-17$ & $\mathrm{~A}, \mathrm{P}$ & 20 & 61,9 & $\begin{array}{l}\text { Arthrocnemum fruticosum (3.3), } \\
\text { Juncus maritimus (3.2) }\end{array}$ \\
\hline 18 & $7 \mathrm{a}$ & $<5$ & $5-12$ & $\mathrm{C}, \mathrm{D}, \mathrm{E}, \mathrm{P}$ & 32 & 56.3 & Juncus maritimus (3.2), Paspalum vaginatu \\
\hline 19. & $7 \mathrm{a}$ & $<5$ & $18-60$ & A,P & 16 & 100 & Scirpus litoralis (3.1), S. maritimus (3.1) \\
\hline 20 & $7 \mathrm{a}$ & $<5$ & $0-40$ & A,P & 18 & 94.4 & $\begin{array}{l}\text { Arthrocnemum fruticosum (3.3), } \\
\text { Cotula coronopifolia (3.4) }\end{array}$ \\
\hline 21 & $7 \mathrm{a}$ & $<5$ & $15-50$ & $A, P$ & 15 & 100 & $\begin{array}{l}\text { Scirpus litoralis }(2.1), \text { S. maritimus }(2.1) \text {, } \\
\text { Cotula coronopifolia (2.3) }\end{array}$ \\
\hline 22 & $7 \mathrm{a}$ & 5 & $0-7$ & $\mathrm{~A}, \mathrm{P}$ & 23 & 82.6 & $\begin{array}{l}\text { Tamarix gallica (2.4), Juncus maritimus (2.2), } \\
\text { Puccinellia sp. (2.3) }\end{array}$ \\
\hline 23 & $7 \mathrm{a}$ & 5 & $0-22$ & $\mathrm{~A}, \mathrm{P}$ & 15 & 86.7 & Juncus maritimus (3.2), Tamarix africana (3.4) \\
\hline 24 & $7 \mathrm{a}$ & $<5$ & 0 & $\mathrm{P}$ & 8 & 87.5 & Tamarix africana $(5.5)$ \\
\hline 25 & $7 \mathrm{a}$ & $<5$ & 20 & E,P & 7 & 100 & Typha angustifolia (5.5) \\
\hline 26 & $7 \mathrm{a}$ & $<5$ & $15-25$ & $\mathrm{C}, \mathrm{P}$ & 6 & 100 & Lemna minor (5.5) \\
\hline 27 & $7 \mathrm{a}$ & $<5$ & $20-60$ & E.P & 4 & 100 & Lemna gibba $(5.5)$ \\
\hline 28 & $7 \mathrm{a}$ & $<5$ & 25 & E,P & 5 & 100 & Phragmites australis (5.5) \\
\hline 29 & $7 \mathrm{a}$ & $<5$ & 0 & $\mathrm{P}$ & 9 & 88.9 & Puccinellia sp. (3.3), Schoenus nigricans (3.2) \\
\hline 30 & $7 \mathrm{~b}$ & $<5$ & $5-20$ & $\mathrm{D}, \mathrm{E}, \mathrm{P}$ & 38 & 76.3 & Sparganium erectum $(5.5)$ \\
\hline 31 & $7 \mathrm{~b}$ & $<5$ & $6-60$ & $\mathrm{E}, \mathrm{P}$ & 22 & 68.2 & $\begin{array}{l}\text { Scirpus maritimus (3.1), } \\
\text { Polypogon monspeliensis (3.1) }\end{array}$ \\
\hline 32 & $8 b$ & $<5$ & 0 & A & 12 & 33.3 & Arthrocnemum fruticosum (4.4) \\
\hline 33 & $11 \mathrm{~b}$ & 10 & 0 & $\mathrm{P}$ & 15 & 60 & Leontodon maroccanus (3.1), Carex divisa (3.1) \\
\hline 34 & $11 \mathrm{~b}$ & 10 & 0 & $\mathrm{C}, \mathrm{P}$ & 14 & 64.3 & Paspalum paspalodes $(5.5)$ \\
\hline 35 & $11 \mathrm{~b}$ & 10 & 0 & A,P & 26 & 57.7 & Juncus inflexus (3.2) \\
\hline 36 & 14 & 1075 & $0-5$ & $\mathrm{P}$ & 15 & 73.3 & Prunus lusitanica (3.4), Ilex aquifoliumum (3.4) \\
\hline 37 & 14 & 700 & $0-50$ & $\mathrm{P}$ & 9 & 88.9 & Juncus effesus (3.2), Ludwigia palustris (3.4) \\
\hline 38 & 14 & 600 & $0-5$ & $\mathrm{P}$ & 14 & 100 & $\begin{array}{l}\text { Baldellia ranunculoides (3.1), } \\
\text { Cyperus flavescens (3.2), C. rotundus (3.1) }\end{array}$ \\
\hline 39 & 15 & $<1$ & $0-65$ & $\mathrm{C}, \mathrm{P}$ & 29 & 72.4 & Typha angustifolia (5.5) \\
\hline 40 & 15 & $<10$ & $0-5$ & $\mathrm{C}, \mathrm{P}$ & 34 & 70.6 & Juncus acutus (5.2) \\
\hline 41 & 17 & 80 & 0 & $\mathrm{P}$ & 15 & 40 & Tamarix gallica (2.4), Nerium oleander (2.4) \\
\hline 42 & $20 \mathrm{a}$ & 300 & 0 & $\mathrm{C}, \mathrm{P}$ & 23 & 60.9 & Salix purpurea (5.5) \\
\hline 43 & $20 \mathrm{a}$ & 300 & 0 & $\mathrm{P}$ & 29 & 69 & Tamarix africana (3.3), T. gallica (3.3) \\
\hline 44 & $20 \mathrm{a}$ & 290 & 0 & $\mathrm{P}$ & 9 & 77.8 & Juncus inflexus (4.2) \\
\hline 45 & $20 \mathrm{c}$ & 300 & $0-42$ & $\mathrm{P}$ & 24 & 54.2 & Salix pedicellata (3.4), Alnus glutinosa (3.4) 46 \\
\hline 21 & 180 & 0 & A & 35 & 45.7 & \multicolumn{2}{|c|}{ Coriaria myrtifolia $(5.5)$} \\
\hline 47 & 23 & 125 & 0 & $\mathrm{~A}, \mathrm{C}, \mathrm{P}$ & 14 & 71.4 & Juncus subulatus (5.5) \\
\hline 48 & 23 & 125 & $0-10$ & A,C,P & 11 & 72.7 & Juncus maritimus (5.2) \\
\hline 49 & 24 & 175 & 35 & $\mathrm{P}$ & 1 & 100 & Potamogeton nodosus (5.5) \\
\hline
\end{tabular}




\begin{tabular}{|c|c|c|c|c|c|c|c|}
\hline $\mathbf{R}$ & $\mathbf{S}$ & Al & $\mathrm{Sb}$ & Aa & Nt & $\mathrm{Nh}$ & Espèces dominantes (ad.s) \\
\hline 50 & 24 & 175 & 0 & $\mathrm{C}, \mathrm{P}$ & 6 & 66.7 & Vitex agnus-castus (3.3) \\
\hline 51 & $25 \mathrm{a}$ & 380 & $0-17$ & $\mathrm{P}$ & 30 & 60 & $\begin{array}{l}\text { Salix pedicellata (2.3), Nerium oleander (2.3), } \\
\text { Fraxinus angustifolia }(2.3)\end{array}$ \\
\hline 52 & $25 \mathrm{a}$ & 425 & 0 & $\mathrm{C}, \mathrm{P}$ & 18 & 33.3 & Nerium oleander $(5.5)$ \\
\hline 53 & $25 \mathrm{a}$ & 425 & 11 & $\mathrm{P}$ & 11 & 100 & Cyperus flavescens (5.5) \\
\hline 54 & 27 & 250 & 0 & $\mathrm{P}$ & 9 & 66.7 & Nerium oleander (5.4) \\
\hline 55 & 28 & 125 & $8-13$ & $\mathrm{P}$ & 30 & 76.7 & Mentha rotundifolia (3.3), Apium nodiflorum (3.3) \\
\hline 56 & $30 \mathrm{a}$ & $<5$ & 40 & $\mathrm{P}$ & 10 & 90 & Potamogeton nodosus (3.4) \\
\hline 57 & $30 \mathrm{a}$ & $<5$ & 0 & $\mathrm{D}, \mathrm{P}$ & 20 & 75 & Juncus acutus (5.2) \\
\hline 58 & $30 \mathrm{a}$ & $<10$ & $0-18$ & $\mathrm{P}$ & 20 & 85 & Cyperus mundtii (5.5) \\
\hline 59 & $30 \mathrm{~b}$ & $<5$ & 0 & $\mathrm{C}, \mathrm{D}, \mathrm{E}, \mathrm{P}$ & 12 & 41.7 & Tamarix gallica $(5.5)$ \\
\hline 60 & $30 \mathrm{~b}$ & $<5$ & 0 & C,D,P & 17 & 58.8 & Juncus acutus (5.2) \\
\hline 61 & $30 \mathrm{c}$ & $<5$ & 45 & $\mathrm{P}$ & 4 & 100 & Paspalum paspalodes (5.5) \\
\hline 62 & $31 \mathrm{a}$ & 156 & $0-7$ & $\mathrm{P}$ & 24 & 37.5 & Salix pedicellata (3.3), Nerium oleander (3.3) \\
\hline 63 & $31 \mathrm{~b}$ & 150 & $0-16$ & $\mathrm{P}$ & 19 & 47.4 & Nerium oleander (3.3), Coriaria myrtifolia (3.3) \\
\hline 64 & 36 & 1460 & $0-7$ & A & 21 & 66.7 & Pteridium aquilinum (3.4), Carex distans (3.2) \\
\hline 65 & 39 & 255 & 0 & $\mathrm{~A}, \mathrm{~B}$ & 21 & 9.5 & Juncus acutus (5.2) \\
\hline 66 & 40 & 755 & $0-23$ & $\mathrm{P}$ & 35 & 65.7 & $\begin{array}{l}\text { Tamarix africana (2.3), Nerium oleander (2.3), } \\
\text { Rubus ulmifolius (2.3) }\end{array}$ \\
\hline 67 & 42 & 40 & $0-20$ & $\mathrm{P}$ & 28 & 28.6 & Tamarix gallica (3.3), Tamarix africana (3.3) \\
\hline 68 & 43 & 75 & $0-15$ & $\mathrm{D}, \mathrm{P}$ & 16 & 62.5 & Tamarix gallica (3.3), Paspalum vaginatum (3.3) \\
\hline 69 & 44 & $<10$ & $5-45$ & PI & 6 & 100 & $\begin{array}{l}\text { Scirpus litoralis (3.3), Potamogeton nodosus (3.3), } \\
\text { Phragmites australis (3.3) }\end{array}$ \\
\hline 70 & 45 & $<10$ & 0 & $\mathrm{~A}, \mathrm{~B}$ & 18 & 38.9 & Popuplus euphratica (3.3), Tamarix gallica (3.3) \\
\hline
\end{tabular}

Table 1. Table synthétique des relevés effectués. $\mathbf{R}, \mathrm{n}^{\circ}$ du relevé. $\mathbf{S}, \mathrm{n}^{\circ}$ de la station (voir Fig. 1). Al, altitude en $\mathrm{m}$. Sb, submersion en $\mathrm{cm}$. Aa, activités anthropozoogènes majeures (A: aménagement (défrichement, assèchement, ...); B: boisement; C: coupe de macrophytes; D: décharge de déchets solides; E: effluent d'eaux usées domestiques; P: pâturage; Pê: pêche). ad.s., indices d'abondance-dominance et de sociabilité. $\mathbf{N h}$, pourcentages d'hygrophytes par relevé. Nt, nombre total d'espèces par relevé. Synthetic table of floristic inventories.

Biologie de la Faculté des Sciences de l'Université Abdelmalek Essaâdi de Tétouan.

\section{RESULTATS ET DISCUSSION}

\section{Diversité spécifique}

Dans cette étude, 389 espèces dont 175 caractéristiques des zones humides ont été identifiées (cf. annexe). Ces dernières correspondent à 89 familles parmi lesquelles, les Graminae, les Cyperaceae, les Juncaceae et les Compositae sont les mieux représentées puisqu'elles correspondent à $27.5 \%$ de l'effectif spécifique global et comprennent $35.84 \%$ des hygrophytes. Bien que les Dicotyledones l'emportent largement sur les
Monocotyledones au niveau global, ces dernières montrent une grande affinité aux zones humides. Certaines espèces ayant une affinité non exclusive aux zones humides comme Agrostis capillaris, Briza maxima, Cynodon dactylon, Elymus elongatus, Holcus lanatus, Lolium multifolim, Polygonum equisetiforme, Clematis vitalba, Crataegus monogyna, Smyrinum olusatrum, Limoniastrum monopetalum, Limonium cymuliferum, L. gummiferum, L. ferulaceum, Bidens pilosa, Pulicularia sicula, Sonchus oleraceus et Suaeda fruticosa, n'ont pas été considérées comme des hygrophytes.

Il importe aussi de citer trois autres phanérophytes marines soulignées récemment par González García (1994) et González García \& Conde Poyales (1995) le long de la côte 
Saidia-Al Hoceima : Zostera marina, Cymodocea nodosa et Posidonia oceanica.

D'une manière générale, on assiste à une grande régression des zones humides suite à l'effet combiné des interventions humaines et des changements climatiques. En effet, l'assèchement des zones humides constitue l'un des principaux facteurs qui menacent la diversité de la flore vasculaire hygrophile autochtone (Stevenson, 1988). L'assèchement est dû à un grand nombre de facteurs dont les principaux sont : le drainage excessif, le pompage des eaux à partir de la nappe phréatique, la décharge incontrôlée de remblais, et la construction de digues et de barrages. La pollution représente également une cause importante de la disparition ou de la raréfaction des hygrophytes (Meriaux, 1982); elle est due principalement aux eaux usées et aux décharges des ordures ménagères. A cet égard, les cas de la Daya Sghira et de la Merja Sidi Kacem/ Tanger constituent des exemples très démonstratifs. En effet, Ionesco et Stefanesco (1967) ont dénombré plus de 50 espèces hygrophiles dont quelques unes étaient décrites comme assez rares. Tandis que le relevé que nous avons effectué à la Daya Sghira (S.2) montre une très forte altération de la composition spécifique puisque sur les 21 espèces du relevé, seules 4 sont hygrophiles.

Par ailleurs, Posidonia oceanica, endémique de la Méditerranée, est en voie de disparition de la lagune de Bou Areg (Nador) après son envahissement par Caulerpa prolifera (González García, 1994 et González García \& Conde Poyales, 1995). Ce problème d'étouffement des phanérogames marines submergées par les algues envahissantes a été signalé dans d'autres régions (Den Hartog, 1994). Les activités portuaires croissantes et les rejets des eaux usées constitueraient donc, à long terme, une menace de la diversité de plusieurs habitats côtiers du Maroc méditerranéen.

De plus, sur le plan local, on peut qualifier, entre autres, plusieurs espèces d'assez rares telles Eryngium corniculatum, Iris pseudacorus, Juncus articulatus (S.6), Carex trinervis (S.11), Juncus subulatus (S.23), J. effusus, Ludwigia palustris (S.14), Utricularia vulgaris (S.15), Cyperus laevigatus (S.30 et 40) et Baldellia ranunculoides (S.22). Eryngium corniculatum et Carex trinervis sont, à notre connaissance, identifiées à l'échelle régionale pour la première fois. D'autres espèces ripicoles sont en forte régression à savoir Vitex agnus-castus, Fraxinus angustifolia, Prunus lusitanica, Ilex aquifolium et Alnus glutinosa. Ainsi, on n'a pas retrouvé les hygrophytes telles que Salix atrocinerea Brot., S. elaeagnos Scop. et Tamarix speciosa Ball. \& Maire citées par De la Torre (1955), ni celles mentionnées par Benabid $(1983,1984)$ à savoir Salix atrocinerea Brot. et Betula celtiberica (B. pubescens Ehrh. ssp. celtiberica (Rothm. \& Vasc.) Rivas-Martinez). Ceci met en relief l'importance de l'impact de ces dernières années de sécheresse et l'exploitation des ripisylves. Toutefois, il faut nuancer cette affirmation car certaines espèces telles que Betula alba (B. pendula Roth) citée par Sauvage (1971) ont des aires très localisées et n'ont pas été prospectées par la présente étude. Cependant, la flore hygrophile s'est 'enrichie' par des espèces introduites comme Populus nigra, P. alba, Salix babylonica et Arundo donax (S.11).

\section{Phytoécologie}

\section{1. Formations végétales}

Du point de vue physionomique, les phytocénoses observées peuvent être classées en:

a) Prairies submergées : elles sont très localisées, hétérogènes et comprennent, en plus de certaines algues parenchymateuses telles que Chara sp., d'autres limnophytes comme Zannichellia palustris, Potamogeton pusillus, Ruppia maritima, R. cirrhosa, Zostera noltii (S.1, 6 et 7); Z. marina, Posidonia oceanica et Cymodocea nodosa (González García, 1994). 
b) Formations submergées partiellement flottantes : elles se composent principalement d'autres limnophytes: Potamogeton nodosus, Potamogeton pectinatus, Callitriche palustris, C. stagnalis, Ludwigia palustris et Ranunculus aquatilis (S.6, 7, 14, 24, 30, 44 et Jbel Zem Zem-Tétouan);

c) Prairies libres flottantes : elles sont constituées exclusivement par Lemna gibba et Lemna minor. A côté de ces pleustons, de minuscules formations mégaloplanctoniques d'Utricularia vulgaris ont été observées dans les parties profondes et calmes du marais de l'Oued Laou/Chaouen (S.15);

d) Roselières : elles occupent principalement la majorité des marais et, parfois, les bords des lacs et des cours d'eau. Elles abritent, selon les conditions stationnelles, toute une série de taxons dominants à savoir : Scirpus litoralis var. genuinus, S. maritimus, Paspalum vaginatum, P. paspalodes, Spartina maritima ssp. stricta, Cyperus mundtii, C. flavescens, Juncus articulatus, Apium nodiflorum, Eryngium corniculatum, Phragmites australis, Iris pseudacorus, Typha angustifolia, Carex distans, Baldellia ranunculoides, Polypogon monspeliensis, Sparganium erectum ssp. neglectum et Cotula coronopifolia;

e) Formations à hygrophytes des zones émergées : elles sont très hétérogènes, herbacées à Juncus acutus, J. maritimus, J. subulatus $f$. salinus, J. inflexus, J. effusus, Schoenus nigricans, Paspalum paspalodes, Carex divisa var. eu-divisa, Leontodon maroccanus, Chamaemelum fuscatum, Puccinellia sp. et Pteridium aquilinum; ou ligneuses souvent ripicoles à Populus euphratica, Tamarix gallica, T. africana, Nerium oleander, Rubus ulmifolius, Coriaria myrtifolia, Salix pedicellata, S. purpurea, Fraxinus angustifolia, Vitex agnus-castus, Prunus lusitanica, Ilex aquilinum et Alnus glutinosa;

f) Sansouires ou enganes : ce sont des formations ligneuses assylvatiques des sols salés à Arthrocnemum fruticosum et/ou Limoniastrum monopetalum; elles forment de vastes étendues aux embouchures de certains oueds (S.1 et 8).

\section{II.2. Groupements végétaux}

L'analyse factorielle des correspondances (AFC) a été effectuée à partir d'un tableau constitué de 70 relevés décrits par seulement 50 espèces dominantes (Tab. 1). En effet pour cette analyse, nous avons retenu uniquement les espèces ayant un indice d'abondancedominance supérieur ou égal à 2. Les trois premiers axes de l'AFC «70 Relevés/50 Espèces dominantes» englobent $26.2 \%$ de l'inertie totale, avec des contributions partielles respectives de $10 \%, 8.3 \%$ et $7.9 \%$. Ces valeurs peuvent apparaître comme relativement faibles; mais sans compromettre le pouvoir discriminant des axes factoriels. En effet, il s'agit d'un fait courant dans l'analyse des tableaux disjonctifs complets à codage logique (Foucart, 1985).

L'examen de la projection du plan factoriel $(1,2)$, donné par la figure 2 , met en évidence l'existence de 5 groupes (A, B, C, D et E).

Le groupe $A$, dont les points sont concentrés autour de l'origine, regroupe 32 relevés. C'est un groupe relativement hétérogène si l'on se réfère au type d'habitat et à la physionomie des groupements. En effet, il rassemble les relevés caractérisés par une seule espèce dominante (Juncus inflexus, J. acutus, Cyperus mundtii, C. flavescens, Paspalum paspalodes, Typha angustifolia, Zostera noltii, Spartina maritima, Potamogeton pectinatus, P. nodosus, Sparganium erectum, Lemna gibba ou L. minor). Il comprend également les groupements peu fréquents et composés d'espèces relativement rares telles que Eryngium corniculatum, Juncus articulatus, $J$. subulatus, J. effesus, Iris pseudacorus, Prunus lusitanica, Ilex aquifolium, Vitexagnus-castus, 


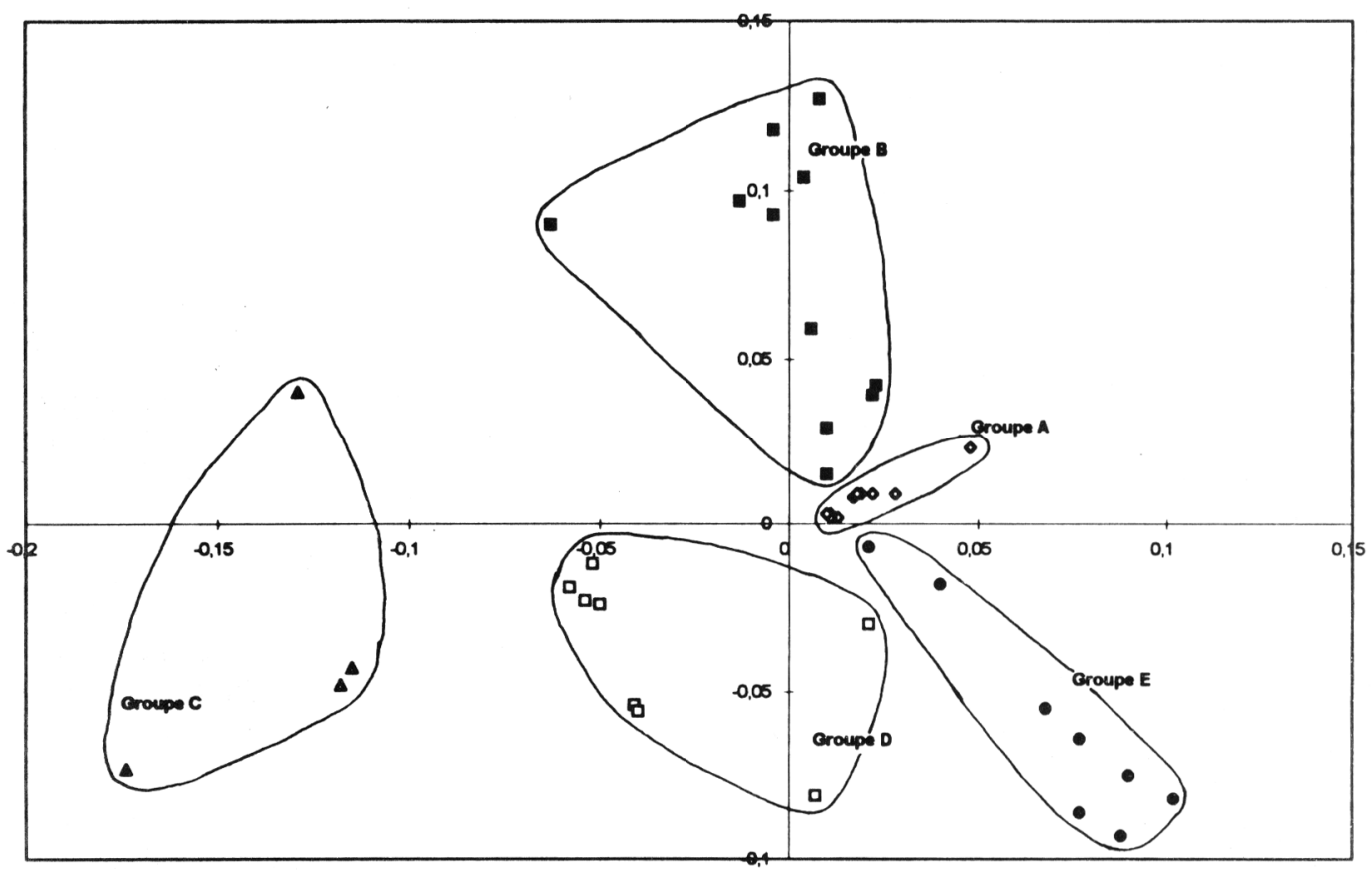

Figure 2. Premier plan de l'AFC « Relevés-Espèces dominantes »; les points cachés n'apparaissent pas. First plan of « Floristic inventories-Dominante species » factorial analysis of correspondences.

ou Ludwigia palustris.

Les quatre groupes restants correspondent à des relevés à dominance bi- ou plurispécifique et recoupent partiellement la classification à partir des formations végétales :

Le groupe B comprend 13 relevés et correspond à des groupements d'embouchures et de marais salés ou saumâtres, herbacés à Puccinellia sp., Juncus maritimus et Schoenus nigricans ou ligneux à Phragmites australis et Arthrocnemum fruticosum, et à durée de submersion inférieure à 6 mois;

Le groupe $\mathrm{C}$ (5 relevés) correspond aux roselières de marais d'eaux douces ou légèrement saumâtres, herbacées à Scirpus maritimus et $S$. litoralis, et à durée de submersion supérieure à 6 mois;

Le groupe D (11 relevés) rassemble les groupements ripicoles ou bordant les marais, d'eaux salées ou saumâtres, ligneux à Tamarix africana, T. gallica et Populus euphratica, et à durée de submersion inférieure à 6 mois;

Le groupe E ( 9 relevés) comporte des ripisylves d'amont, des eaux douces, à Nerium oleander, Salix pedicellata, Coriaria myrtifolia, Fraxinus angustifolia, Rubus ulmifolius ou Alnus glutinosa; et à durée de submersion inférieure à 6 mois.

\section{II.3. Synécologie}

Plusieurs facteurs, dont l'intensité peut varier considérablement, agissent mutuellement sur le développement des hygrophytes. Ainsi, on a mis en évidence que plus la diversité spécifique à l'échelle des groupements végétaux de zones humides est faible, plus la salinité, la fertilité et/ou l'humidité du sol sont importantes (Lachavanne, 1982; Shaltout et al., 1995). Dans ces conditions extrêmes, l' apparition d'espèces hautement dominantes peut être due à la 


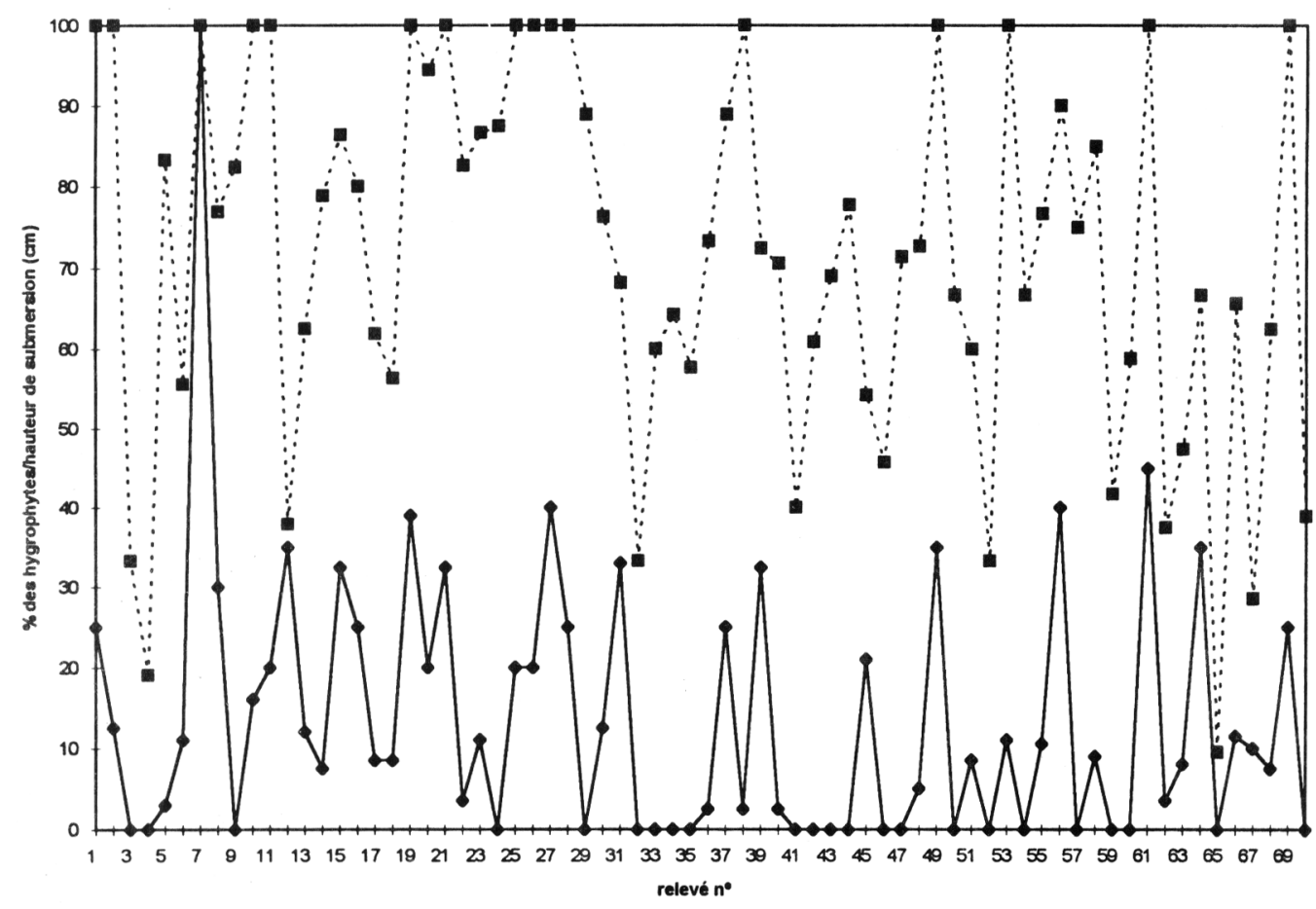

Figure 3. Fluctuation de la hauteur de submersion et du pourcentage des hygrophytes en fonction des relevés. Wetland species percentage and submersion depth fluctuation by floristic inventory.

suppression des espèces les moins compétitives. Les perturbations de l'habitat peuvent avoir la même conséquence (Shaltout et al., 1995). Concernant cette étude, la submersion, la salinité, la charge organique des eaux usées, le mésoclimat et les travaux d'aménagement constituent les principaux facteurs.

\section{II.3.1. Submersion}

La performance des macrophytes est très influencée par l'hydrologie du milieu (in Atbib, 1979; Breen et al., 1988; Gosselink \& Turner, 1978; Naomi \& Ganf, 1994). La principale contrainte imposée par la submersion est l'empêchement des échanges gazeux dans la mesure où la résistance à la diffusion de la plupart des gaz est approximativement dix milles fois plus grande dans l'eau que dans l'air (Armstrong et al., 1994). Le comportement envers l'hydromorphie édaphique se reflète très bien chez les espèces dominantes puisqu'elles présentent une dominance écologique et physiologique (Cain \& Castro, 1959).

La corrélation entre la présence des hygrophytes, représentée par le pourcentage de ces dernières dans les relevés, et la submersion montre l'importance du pouvoir sélectif de ce paramètre (fig. 3). En effet, les pourcentages les plus élevés sont généralement associés à des relevés effectués dans des stations caractérisées par des hauteurs de submersion importantes, sauf quelques exceptions où d'autres facteurs sont plus déterminants. En effet, le coefficient de corrélation $(r=0.47)$ entre le pourcentage d'hygrophytes et la submersion est significatif au seuil de 0.001 .

De plus, les espèces dominantes ne montrent pas la même amplitude écologique 
vis-à-vis de la hauteur de submersion. Ainsi, la classe la plus représentée est celle des espèces à submersion nulle ou faible $(0<\mathrm{h}<10 \mathrm{~cm})$. Elle est suivie par les classes d'espèces à submersion moyenne $(10<\mathrm{h}<20 \mathrm{~cm})$, forte $(20<\mathrm{h}<30 \mathrm{~cm})$ ou très forte $(\mathrm{h} \geq 30 \mathrm{~cm})$. Cependant, d'autres espèces peuvent tolérer de grands intervalles de variations qui leur confèrent une grande plasticité écologique envers ce facteur (tab. 2).

Les perturbations liées aux travaux d'aménagement (installation de digues, évacuation des eaux usées, ...) entraînent, suivant les cas, un assèchement (S.6, 7, 21 et 39) ou, au contraire, une humidification (S.3 et 7), qui, en modifiant l'état de submersion, peuvent changer la composition floristique de plusieurs sites humides. Dans les zones humidifiées, où les hélophytes résistent à des variations bien déterminées de la profondeur d'eau, ce sont les plantes flottantes qui dominent en premier lieu (Ranunculus aquatilis, Lemna sp.); les plantes submergées, ayant des difficultés d'ajustement aux variations de la profondeur (in Stevensen, 1988), ne dominent que lorsque le niveau d'eau se stabilise; c'est le cas du Potamogeton pectinatus (S.6) et de Ruppia maritima (S.8). Le contraire s'observe à l'embouchure artificielle de l'oued Negro où les niveaux d'eau subissent des fluctuations considérables; on assiste seulement à un développement de petites touffes de Ruppia cirrhosa aux bords.

La nature des espèces végétales occupant les sites asséchés, varie suivant l'importance de la durée du retrait des eaux. Un site immergé, une fois exondé, est peuplé par des espèces telles que Crypsis aculeata qui supportent une légère humidité superficielle et d'autres espèces comme Cotula coronopifolia qui tolèrent une très faible immersion (S.7); dans d'autres sites, ce sont Leontodon maroccanus et Plantago coronopus ssp. coronopus qui prennent le relais (S.11). Pulicaria arabica marque l'état de post-submersion surtout dans les marais temporaires (S.6) comme il a été souligné aussi bien chez cette espèce que chez Spergularia purpurea et Tillaea vaillantii par Nègre (1956) aux Dayas de Jbilete-Elkelaâ Srarhna/Maroc. Dans le cas où la période d'émergence peut dépasser largement six mois, ce sont les hygrophytes des zones émergées qui s'installent (S.7).

Du point de vue de la dynamique de la végétation, les hélophytes entièrement émergées pendant l'été ne marquent qu'un stade intermédiaire de la succession végétale. Tandis que les phanérophytes, dont le système racinaire est plus profond, constituent le stade climacique qui correspond dans ces cas à une forêt humide. Irvine \& West (1979), Everitt (1980) et Petranka \& Holland (1980) rapportent que l'élimination de la submersion favorise l'envahissement du Tamarix dans l'ouest des USA (in Brock, 1994). Nos propres observations au niveau de certains sites confirment ces constations, spécialement dans le cas de certaines embouchures (S.3). Un cas similaire est observé actuellement à la Merja Sidi Boughaba par extension de la Juniperaie à Juniperus phoenicia (Benabid, communication personnelle).

\section{II.3.2. Salinité}

La répartition des zones humides salines (lagune, estuaire, sebkha et marais salants) dans le secteur d'étude, est liée essentiellement aux influences marines. En effet, il est communément admis qu'aussi bien l'intensité que la fréquence du mélange entre les eaux continentale et marine entraînent des modifications importantes des caractéristiques chimiques de l'eau et des populations biologiques qui y habitent (Ketchum, 1954; in Comín et al., 1987).

Mise à part la végétation hygrophile se développant dans des sites où le seuil de sensibilité des espèces à la salinité n'est pas atteint, on a identifié des groupements à 


\begin{tabular}{l|c|c|c|c}
\hline Formation à & \multicolumn{4}{|c}{ Hauteur de submersion $(\mathrm{cm})$} \\
\cline { 2 - 5 } & {$[0-10[$} & {$[10-20[$} & {$[20-30[$} & $\geq 30$ \\
\hline
\end{tabular}

\section{Juncus maritimus}

Juncus acutus

Puccinellia sp.

Tamarix gallica

Tamarix africana

Schoenus nigricans

Leontodon maroccanus

Juncus inflexus

Juncus effesus

Carex distans

Cotula coronopifolia

Pteridium aquilinum

Arundo donax

Arthrocnemum fruticosum

Limoniastrum monopetalum

Juncus subulatus

Carex divisa

Cyperus rotundus

Baldellia ranunculoides

Eryngium cornicultum

Juncus articulatus

Iris pseudacorus

Paspalum vaginatum

Polygonum persicaria

Sparganium erectum

Potamogeton nodosus

Apium nodiflorum

Mentha rotundifolia

Cyperus flavescens

Cyperus mundtii

Scirpus litoralis

Scirpus maritimus

Potamogeton pectinatus

Ruppia maritima

Typha angustifolia

Ludwigia palustris

Spartina maritima*

Phragmites australis

Zostera noltii*

Paspalum paspalodes
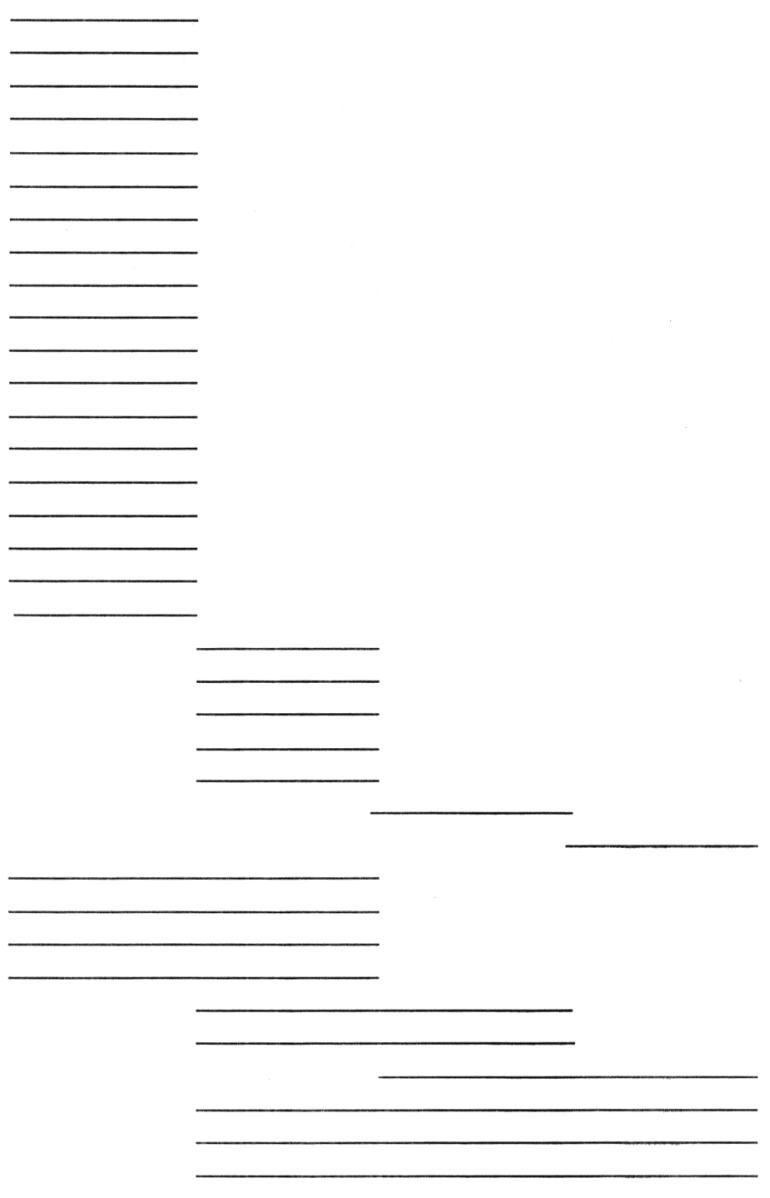

Table 2. Classification simplifiée des principales formations en fonction de la hauteur de submersion utilisant les données des sites à régime hydrique non perturbé. (* Espèces d'embouchures obéissant au régime marin). Simplified classification of the chief species beds in terms of the submersion depth using data of undisturbed-hydrology sites.

halophytes strictes à Arthrocnemum fruticosum, Limoniastrum monopetalum, Spartina maritima ou Zostera noltii (S.1 et 8), et des groupements mésohalophiles à Juncus acutus,
J. subulatus, J. maritimus, Puccinellia sp., Schoenus nigricans, Tamarix gallica, $T$. africana, Phragmites australis, Potamogeton pectinatus, Ruppia maritima, $R$. cirrhosa ou 
Populus euphratica (S.6, 7, 23, 30, 42 et 45). Au bord de la sebkha Bou Aerg (Kariet Akermane/Nador), on a noté la cohabitation de Phragmites australis et Arthrocnemum fruticosum en formant des groupements quasibispécifiques. Cette observation confirme la grande plasticité écologique du roseau commun vis- à-vis de la salinité (Gorenflot et al., 1984; Tahiri, 1987; Zrid, 1988).

Des conclusions similaires aux nôtres ont été relevées par différents auteurs, chez Potamogeton pectinatus (Haslam, 1978; Van Wijk, 1988; Verhoven, 1980; De Thioux, 1989), Ruppia cirrhosa (Meriaux, 1982), Juncus subulatus (Rivaz Martinez et al., 1980), J. maritimus (Benchâabane \& Abbad, 1995), Schoenus nigricans (Vanden Berghen, 1972) et Populus euphratica (Boukil, 1986). D'autre part, Chapman (1960) affirme que Juncus acutus présente une faible tolérance tout en restant associée à de bonnes conditions de drainage.

En ce qui concerne les formations ripicoles, le Tamarix représente le genre dominant des ripisylves côtières (S. 6, 20, 30, 42, 43 et 45). En amont des oueds, d'autres espèces telles que Nerium oleander, Rubus ulmifolius et certaines espèces caducifoliées, moins résistantes à la salinité, peuvent entrer en compétition avec le Tamarix ( $\mathrm{S} .16$ et 40 ). Cependant, ce dernier peut conserver son caractère dominant si les conditions de salinité ne changent pas (S.42). Fernandez Gonzalez et al. (1990) précisent à ce propos que sous climat aride où l'effet de la salinisation est fréquent, la végétation du Tamaricion africanae remplace les ripisylves de Salicitalia purpureae et Populetalia. Les ripisylves à Nerium oleander abondent exceptionnellement dans l'embouchure de l'oued Oûrînga/El Jebha à l'opposé de Ras Al Kala (embouchure de l'oued Beni Boufrah), oueds Smir et Negro/Tétouan. Ceci est vraisemblablement dû à l'envahissement des nappes alluviales par les eaux salines qui défavorisent le développement de cette espèce aux embouchures des oueds.

Dans certains sites comme les marais de l'oued Smir, on observe une accentuation de la salinisation, d'origine relativement récente, et cela suite à une forte perturbation du milieu. En effet, la construction d'un barrage en amont et d'un port de plaisance à l'embouchure ont profondément modifié les équilibres hydriques d'une part, en limitant les apports en eau douce et d'autre part, en facilitant l'entrée des eaux marines. Des études récentes montrent une augmentation de la salinité de la nappe suite à l'invasion des eaux marines et une accentuation de la salinité édaphique (Chellaf, 1990; Stitou \& Pulido-Bosch, 1995). En conséquence, c'est la salicornaie qui se développe au détriment de la scirpo-junçaie (S7) de la bordure de la lagune. D'autre part, dans les niveaux relativement élevés du marais Smir, des espèces indicatrices de salinité, comme Spergularia marina, Limonium ferulaceum et Arthrocnemum fruticosum, s'installent sous forme isolée et sporadique (S.7). L'assèchement d'autres zones, dû à la mobilisation des eaux et/ou au comblement partiel des embouchures, peut susciter le développement d'autres espèces halophiles telles que Frankenia laevis et $F$. corymbosa (S.6 et 39 ).

D'autre part, les effluents domestiques bruts par l'effet d'une simple dilution, facilitent le développement dans des zones humides salées, des typhaies, scirpaies, etc. (S.3 et 7).

\section{II.3.3. Charge organique domestique}

Dans les zones humides marquées par une très grande pression humaine, on retrouve les espèces végétales rudérales ou nitrophiles telles que Anthemis cotula, Nicotiana glauca, Datura stramonium, Solanum nigrum, Chenopodium murale, C. album, C. vulvaria, Echinochloa crus-galli, Setaria verticellata, Raphanus raphanistrum et Ricinus communis (S.6, 7, 14, 16, 20 et 43).

En outre, l'eutrophisation cause de graves 
problèmes dans les écosystèmes aquatiques des eaux douces. Les espèces végétales eutrophisantes se comportent différemment selon le degré de pollution et d'autres facteurs stationnels comme la transparence de l'eau, la température, l'ensoleillement, ... En effet, le comblement partiel des embouchures associé à d'éventuels effluents domestiques, entraîne l'eutrophisation des bras morts isolés (S.6 et 8). Potamogeton pectinatus, Ruppia maritima et les algues filamenteuses constituent alors les principales espèces eutrophisantes de ces sites. Ailleurs, des effets pareils d'eutrophisation ont été notés chez Potamogeton pectinatus et les Chraceae (Haslam, 1978; de Thioux, 1989; Krause \& King, 1994).

Dans le cas des eaux à très faible transparence, ce sont les espèces végétales trouvant accès à la lumière (Lemna sp., Typha angustifolia, Sparganium erectum, etc.), qui prennent le relais (S.3, 7 et 43). D'ailleurs, il a été déjà démontré que les plantes submergées sont fortement limitées par les radiations solaires, d'où l'importance de la profondeur de la couche d'eau qui débilite la pénétration de la lumière ainsi que de la turbidité du milieu qui l'intercepte (Haslam, 1978; Evans et al., 1986; in Stevensen, 1988; Menéndez \& Comín, 1989).

Concernant la résistance des hygrophytes à la charge organique, on peut distinguer les catégories suivantes:

a) les espèces des sites fortement pollués (recevant des effluents bruts d'eaux usées) : Lemna gibba (S.7 et 43), Typha angustifolia (S.3 et 7), Sparganium erectum (S.7) et Polygonum lapathifolium (S.7 et 8);

b) les espèces des sites pollués : Lemna minor (S.6 et 7) et Phragmites australis (S.7);

c) les espèces des sites moyennement à non pollués : Scirpus maritimus (S.6 et 7) et Scirpus litoralis (S.6 et 7), Polypogon monspeliensis (S.1, 3, 6, 7, 15, 31, 40 et 42) et Apium nodiflorum (S.7, 14, 15, 25, 28 et 43).
Par ailleurs, il importe de noter certaines observations concernant la végétation des sites pollués. Ainsi, Polygonum lapathifolium se développe dans des conditions d'immersion très contrastées; elle est omniprésente le long des canaux à ciel ouvert des eaux usées et dans des sites émergés ou immergés, en présence ou non de déchets solides. Des études menées sur les lentilles d'eau montrent que Lemna gibba domine dans les eaux eutrophisées, alors que le contraire est observé chez Lemna minor qui prospère dans des milieux pauvres en nutriments (Rejmankova, 1975; Pérez-Raya \& Lopez-Nieto, 1991; Rivaz-Martinez et al., 1980). A cet égard certains auteurs parlent de la «transformation» de Lemna minor en Lemna gibba lorsque les conditions deviennent défavorables (De Lange \& Westinga, 1979; Landolt, 1975). Dans d'autres régions géographiques, Lemna minor est considérée comme espèce des aires polluées (Haslam, 1978).

De plus, Hejny et Husák (1978) soulignent que les associations Phragmetetum communis (Gams 1927) Schmale 1939 et Typhetum angustifoliae (Allorge 1922) Schmale 1939 sont typiques des étangs littoraux et affectionnent des habitats mésotrophes à eutrophes (in Hroudová \& Zákravsky, 1988). Perez-Raya et Lopez-Nieto (1991) ont rapporté que l'association Junco subnodulosisparganietum erecti Martinez Parras \& Peinado 1983, dominée par Sparganium erectum, se développe généralement sur des sols riches en matière organique. D'ailleurs, en étudiant la productivité souterraine de $S$. erectum, la présence systématique des rhizomes de Scirpus sp. dans les carottes du sol et la faible représentativité de ce genre dans cette station (S.7), prouvent que la scirpaie occupait cette station; et c'est après l'évacuation des eaux usées que la sparganaie s'est installée (Ennabili \& Ater, 1995).

D'autre part, les hygrophytes de sites émergés paraissant résister à la pollution 
domestique se limitent à l'hélophyte Juncus maritimus (S.7) et aux espèces ligneuses ripicoles autochtones telles que Nerium oleander, Tamarix gallica, T. africana et Salix purpurea (S.11 et 43) ou introduites comme Arundo donax, Salix babylonica, Populus nigra et Populus alba (S.11).

\section{II.3.4. Mésoclimat}

Les zones humides naturelles sont généralement localisées sur la frange littorale ayant un macroclimat semi-aride et elles abritent une végétation adaptée plutôt à des mésoclimats voire des microclimats particuliers. Ainsi, Salix pedicellata, Alnus glutinosa, Fraxinus angustifolia et Coriaria myrtifolia forment des groupements affectionnant surtout les ripisylves des vallées humides ( $\mathrm{S} .20,25$ et 31 ) où l'humidité atmosphérique est assez élevée. Certaines espèces peuvent, ainsi, engendrer de petites formations d'ubacs telles que le groupement à Carex distans et Pteridium aquilinum (S.36) et celui à Coriaria myrtifolia (S.21); la strate muscinale y est bien développée. Davallia canariensis constitue de véritables tapis sur l'oléastre et le chêne liège en altitude à Jbel Haouz et Jbel Zem Zem/Tétouan. Sous des climats plus secs, ce sont les Tamarix qui abondent comme l'a mentionné FernandezGonzalez et al. (1990). Toutefois, le roseau commun est indifférent à ces contraintes climatiques puisqu'il peut se présenter, à l'échelle du Maroc, dans tous les étages bioclimatiques (Gorenflot, 1986; Zrid, 1988).

Au sein des formations des vallées humides, l'éclairement relatif associé à d'autres facteurs tels que l'humidité édaphique et la microtopographie favorisent le développement de plusieurs espèces ombrophiles comme Selaginella denticulata, Adiantum capillusveneris, Potentilla micrantha, Pteridium aquilinum, Ruscus hypohyllum, Asplenium adiantum-nigrum et Blechnum spicant (S.14, 21, 25 et 36).

\section{CONCLUSIONS ET PERSPECTIVES}

Cette étude constitue une contribution à l'évaluation de la diversité spécifique de la flore hygrophile du Maroc méditerranéen. Tout en identifiant des espèces rares, elle met en évidence deux espèces citées pour la première fois à l'échelle régionale (Eryngium corniculatum et Carex trinervis). Quant à la synécologie des divers groupements hygrophiles reconnus, elle souligne l'importance des perturbations des sites étudiés (assèchement, pollution liquide domestique et travaux d'aménagement) et du climat sur leur répartition et leur dynamisme. Les différentes approches mettent en exergue une grande richesse spécifique des formations et des groupements végétaux diversifiés et adaptés aux différents types de biotopes.

D'une manière générale, les zones humides ont une valeur immense et accomplissent des fonctions d'une extrême importance. En effet, et à titre d'exemples, elles stabilisent les côtes et les rivages, protègent le littoral, diminuent les risques d'inondations, tamponnent le microclimat.. etc. Cette liste est loin d'être exhaustive et d'autres fonctions peuvent être valorisées. En effet, plusieurs macrophytes de ces zones sont utilisées dans le traitement des eaux usées dans plusieurs régions du monde (Blake \& Dubois, 1982; Vuillot \& Boutin, 1987; Radoux \& Kemp, 1988; Brix \& Schierup, 1989; Brix, 1994; Braun, 1995). Au Maroc, les approches de ce type sont encore limités et concernent seulement les systèmes à lentilles d'eau (Lemna gibba L.), à roseau ou à jacinthe d'eau (Eichornia crassipes (C.F.P. Mart) SolmsLaub.) (ONEP, 1988; Mandi et al., 1992, 1993; Mandi, 1994; Azzaoui \& Nejmeddine, 1994).

En revanche, la jacinthe d'eau $(E$. crassipes), espèce tropicale et subtropicale, est déconseillée dans notre région principalement à cause de ses capacités d'envahissement des systèmes aquatiques 
locaux (in François, 1979; ONEP, 1988; in Brix \& Schierup, 1989) et des pertes d'eau par évapotranspiration qui peuvent atteindre $60 \%$ en été sous climat aride (Mandi et al., 1992, 1993). De surcroît, ces techniques d'épuration appelées souvent zones humides reconstituées demeurent intransférables d'une région géographique à une autre (Brix \& Schierup, 1989; Radoux et al., 1995). Il en résulte que les groupements des macrophytes des sites pollués, mis en évidence dans ce travail, ont un grand intérêt pour une éventuelle utilisation dans le traitement des eaux usées. D’ailleurs, parmi les scénarios d'épuration décentralisée des eaux usées de Tétouan (NW du Maroc), les systèmes marécageux figurent à côté du lagunage naturel aéré (BRYLER-ISKANE-SHELADIA, 1992).

\section{ANNEXE}

Liste des espèces strictement hygrophiles déterminées : Fl. Af. N., Flore de l'Afrique du Nord (Maire 1952/1982); Fl. Al., Nouvelle Flore de l'Algérie et des Régions Désertiques Méridionales (Quézel \& Santa 1962/1963). Les stations de présence de chaque espèce sont indiquées entre parenthèses

Adiantum capillus-veneris (15, 21, 25, 31 et Jbel Haouz-Tétouan); Aeluropus littoralis (6); Agrostis canina (28); Alisma plantago-aquatica (3, 7 et 15); Alnus glutinosa $(14,20$ et 21); Anagallis crassifolia (14); Apium nodiflorum (3, 7, 14, 15, 20, 25, 28, 30, 31 et 43); Arundo donax (7, 11, 25 et 40); Arthrocnemum fruticosum (1, 7, 8, 42 et 45); Arthrocnemum perenne (1, 3 et 7); Asplenium adiantum-nigrum (14 et Jbel Haouz-Tétouan); Asplenium ceterach (Jbel Haouz-Tétouan); Asplenium trichomanes ( Jbel Haouz-Tétouan); Atriplex portulacoides (1); Baldellia ranunculoides (11,14 et 22); Blechnum spicant (14); Briza minor (22); Callitriche palustris (7); Callitriche stagnalis (Jbel Zem Zem-Tétouan); Carex acuta (20,21, 25 et 31); Carex distachya (3, 6 et 7); Carex distans (7, 11 et 36); Carex divisa (2, 3, 7 et 11); Carex echinata (20); Carex helodes (11); Carex muricata
(15); Carex serotina (8); Carex trinervis (11); Catabrosa aquatica (11); Centaurea diluta (8); Centaurium pulchellum $(3,6,7,11,15,17,25,40$ et 45); Centaurium spicatum $(6,7,11,15,23,30,40$ et 45); Chamaemelum fuscatum (11 et 25); Chenopodium chenopioides (30); Coriaria myrtifolia (21 et 31); Cotula coronopifolia (3, 6 et 7); Crataegus laciniata (36); Crypsis aculeata (7 et 23); Cyperus esculentus (11); Cyperus flavescens (14 et 25); Cyperus fuscus (28 et 30); Cyperus laevigatus (30, 40 et Koudiat Taiffour-Tétouan); Cyperus longus (6, 7 et 20); Cyperus mundtii $(6,14$, 15 et 30); Cyperus rotundus (14, 20 et 30); Damasonium alisma (3, 6 et 7); Daucus carota (3); Davallia canariensis (Jbel Haouz et Jbel Zem ZemTétouan); Dipsacus fullonum (3); Dorycnium rectum (3 et 20); Dryopteris filix-mas (Jbel Haouz-Tétouan); Echinochloa crus-galli (6); Eleocharis palustris (3, 6,7 et 15); Eleocharis quinqueflora (22); Epilobium parviflorum (6, 15 et 30); Epilobium tetragonium (21); Equisetum ramosissimum (25, 28, 30 et 40); Equisetum telmateia (25); Eryngium corniculatum (6); Eupatorium cannabinum (25); Euphorbia pubescens (40); Festuca pratensis (6 et 7); Fraxinus angustifolia (14 et 25); Geranium dissectum (39); Gomphocarpus fruticosus (15 et Jbel HaouzTétouan); Hainardia cylindrica (3); Hordeum hystrix (3); Hordeum murinum (7); Ilex aquifolium (14); Indigofera articulata, in FI. Al. (21 et 25); Inula crithmoides (7 et 45); Iris pseudacorus (6 et Jbel Haouz-Tétouan); Juncus acutiflorus (3); Juncus acutus $(3,6,7,8,15,23,30,39,40,42,43$ et 45); Juncus articulatus (6); Juncus bufonius (3, 7 et 21); Juncus bulbosus (14, 28 et 30); Juncus effusus (14, 20 et 36); Juncus fallax, in Fl. Af. N. (3); Juncus inflexus (3, 6, 11 et 20); Juncus maritimus (2, 3, 6, 7,8 et 23); Juncus striatus $(14,15,28,36$ et 40$)$; Juncus subulatus $(6,7,23$ et 40); Laurus nobilis (22 et Belouazene-Tétouan); Lavatera trimestris (15); Lemna gibba (7 et 43); Lemna minor (6, 7, 30 et Jbel Zem Zem-Tétouan); Leontodon maroccanus (11); Lippia nodiflora (15 et 30); Lonicera biflora (40); Lotus palustris (36); Lotus subbiflorus (6 et 7); Ludwigia palustris (14); Lycopus europaeus (3, 6, 7, 14, 15 et 20); Lythrum junceum $(3,6,7,11,15$, 20, 25, 30, 40, Jbel Zem Zem et Koudiat TaifourTétouan); Lythrum tribracteatum (14 et 20); Mentha pulegium $(2,6,7,14,15,17,20,21,23,25,28,30$ et 36); Mentha rotundifolia (7, 14, 15, 20, 21, 25, 27, 28, 30, 31, 36, 40 et 43); Nasturtium officinale 
(30 et Jbel Haouz-Tétouan); Nerium oleander (3, $15,17,20,21,24,25,27,28,30,31,40$ et 43); Ophioglossum lusitanicum (Jbel Zem Zem-Tétouan et forêt diplomatique-Tanger); Osmunda regalis (14); Panicum repens $(6,7,11,28$ et 30); Paspalum paspalodes $(7,11,20$ et 30); Paspalum vaginatum $(6,7,15,20,28,43$ et 44); Pholiurus incurvus (7); Phragmites australis (6, 7, 11, 15, 23, 42 et 44); Plantago major $(6,7,11,20,28,30$ et 40); Polygonum lapathifolium (7, 8, 15, 20, 30 et 40); Polygonum persicaria (14 et 28); Polypodium vulgare (14 et Jbel Haouz-Tétouan); Polypogon monspeliensis $(1,3,6,7,11,15,20,23,31,40$ et 42); Polystichum aculeatum (14); Populus alba (11, 12 et 40); Populus euphratica (45); Populus nigra (11 et 40); Potamogeton nodosus (15, 24, 30 et 44); Potamogeton pectinatus (6 et 44$)$; Potamogeton polygonifolius (22); Potamogeton pusillus (7); Potentilla micrantha (36); Prunus cerasifera (14); Prunus lusitanica (14); Pteridium aquilinum (14, 17, 25, 36 et Jbel Haouz-Tétouan); Puccinellia sp. (6 et 7); Pulicaria arabica (6, 28, 42 et 43); Pulicaria filaginoides, in $\mathrm{Fl}$. Al. (20 et 23); Ranunculus acris (7 et 11); Ranunculus aquatilis (7); Ranunculus arvensis (7 et 11); Ranunculus ficaria (Jbel HaouzTétouan); Ranunculus macrophyllus (7 et Jbel Zem Zem-Tétouan); Ranunculus muricatus (7); Ranunculus sphaerospermus (7); Ranunculus trilobus (3, 6 et 7); Rosa sempervirens (15, 20, 21, 25 et 27); Rubus ulmifolius (3, 15, 17, 20, 21, 24, 25, 27, 31, 36 et 40); Rumex algeriensis, in Fl. Af. N. (6); Rumex bucephalophorus (6 et 7); Rumex conglomeratus $(3,7,15,20$ et 28); Rumex crispus (6, 7 et 36); Rumex obtusifolius (20); Rumex palustris (7); Rumex pulcher (6 et 7); Ruppia cirrhosa (6); Ruppia maritima (6, 7 et 8); Salix alba (6 et 11); Salix babylonica (11 et 12); Salix pedicellata (14, 20, 21, 24, 25, 31 et 36); Salix purpurea (11 et 20); Samolus valerandi $(7,14,15,20,21,25,28,30,31$, 40 et 43); Schoenus nigricans (7, Cap Spartel-Tanger et Jbel Zem Zem-Tétouan); Scirpus cernuus (6, 7 et 28); Scirpus holoschoenus (3, 15, 20, 21, 27, 30, 31 et 40); Scirpus litoralis (6, 7 et 44); Scirpus maritimus (3, 6 et 7); Scrophularia sambucifolia (36); Scrophularia umbrosa (11 et Jbel HaouzTétouan); Selaginella denticulata (21 et 26); Setaria pumila (11); Sonchus maritimus (28 et 30); Spartina maritima (1); Sparganium erectum (6 et 7); Suaeda maritima (1); Tamarix africana $(3,6,7,20,21,40$ et 42); Tamarix canariensis (30); Tamarix gallica
$(6,7,8,16,17,20,21,30,42,43$ et 45); Trachelium caeruleum (21 et 25); Trifolium isthmocarpum (2 et 6); Typha angustifolia (3, 6, 7, 14, 15, 20, 28, 30, 40, 43 et 44); Utricularia vulgaris (15); Veronica anagallis-aquatica (20, 28 et 30); Vitex agnuscastus (5 et 24); Zannichellia palustris (7); Zostera noltii (1).

\section{RÉFÉRENCES BIBLIOGRAPHIQUES}

ACHHAL, A, M. BARBERO, A. BENABID, O. M'HIRIT, C. PEYRE, P. QUÉZEL \& S. RIVASMARTÍNEZ -1980- A propos de la valeur bioclimatique et dynamique de quelques essences forestières au Maroc. Ecol. Mediter., 5: 211-249, Marseille.

ARMSTRONG, W., R. BRÄNDLE \& M.B. JÄKKSON -1994- Mechanisms of flood tolerance in plants (Review). Acta Bot. Neerl., 43 (4), 307-358.

ATBIB, M. -1979- Etude phytoécologique de la réserve biologique de Mehdia (littoral atlantique du Maroc), 1-La végétation hygrophile de la Merja Sidi Bou Ghaba. Bull. Ins. Sci., Rabat, 4, 99-188.

AZZAOUI, B. \& A. NEJMEDDINE -1994- Etude de l'élimination des métaux lourds $(C d, Z n)$ des eaux usées par ruissellement sur roselière. Comm. Coll. Internationale sur le thème : Eau et Pollution, Fac. Sc. Agadir, Maroc.

BCEOM-SECA -1994- Projet Etude et Plans de Gestion des Aires Protégées du Maroc. Bureaux d'Etudes, Montpellier, Rapport Préliminaire, 11, Tome 1, vol. 2 (201p) et vol. 3 (165p).

BENABID, A. -1982-Etudes phytoécologique, biogéographique et dynamique des associations et séries sylvatiques du Rif occidental (Maroc). Thèse Doc. es-Sc., Univ. Aix-Marseille III, Saint-Jérôme.

BENABID, A. -1983- Etudes biogéographique et dynamique des peuplements forestiers du rif (Maroc). Ann. Rech. Forestière au Maroc, 23 : 49-129.

BENABID, A. -1984- Etude phytoécologique des peuplements forestiers et préforestiers du Rif centro-occidental (Maroc). Travaux Inst. Sci., Série Bot., 34, Rabat.

BENCHÂABANE, A. \& A. ABBAD -1995- Les formations végétales d'un milieu halomorphe de la région de Tensift (lac Zima, Chemaia, 
Maroc). Rev. Rés. Amélior. Prod. Agr. Milieu Aride, $7:$ 73-86.

BLAKE, G. \& J.P. DUBOIS - 1982-L'épuration des eaux par les plantes aquatiques. Association Française pour l'Etude des Eaux, CDIE, Paris, $103 \mathrm{p}$.

BOUKIL, A. -1986- Etude et cartographie des groupements végétaux et des possibilités de reboisement dans la lagune de Oued Smir dans un but d'aménagement touristique. Cent. Reg. Et. Am. Tét., Maroc., 19 pp.

BRAUN, M. -1995-L'épuration des eaux usées par lagune à Lemna. N. S. T., Vol. 13, 1, 261-267.

BRAUN-BLANQUET, J. -1968- L'école phytosociologique zurich-montpelliéraine et la SIGMA. Vegetatio, 16:1-78.

BREEN, C.M., K.H. ROGERS \& P.J. ASHTON 1988-Vegetation processes in swamps and flooded plains. in "J-J. Symoens (ed.) : Vegetation of Inland Waters", Kluwer Academic Publishers, Dordrecht, 223-246.

BRIX, H. -1994- Functions of macrophytes in constructed wetlands. Wat. Sc. Tech., 29 (4), $71-78$.

BRIX, H. \& H.-H. SCHIERUP -1989-The use of aquatic macrophytes in water-pollution control. Ambio., 18 (2): 100-107.

BROCK, J-H. -1994- Tamarix spp. (Salt Cedar), an Invasive Exotic Woody Plant in Arid and Semiarid Riparian Habitats of Western USA. in "LC. de Waal, L-E. Child, P-M. Wade \& J-H. Brock (eds.): Ecology and Management of Invasive Riverside Plants", J. Wiley \& Sons Ltd., 27-44.

BRYLER-ISKANE-SHELADIA (Groupement d'Etude) -1992- Etude du schéma directeur d'assainissement liquide de la ville de Tétouan, synthèse de la mission A; Municipalité de Tétouan, Maroc, $62 \mathrm{p}$.

CAIN, S.A. \& G.M. de O. CASTRO -1959-Manual of vegetation analysis. Harper, New York.

CHAPMAN, N-J. -1960- Mediterranean and australasian salt marshes. Eurasian salt deserts. in "Salt Marshes, and Salt deserts of the World", Leonard Hill Intersciences, 205-237.

CHELLAF, A. -1990-Cartographie semidétaillée des sols de Tétouan, échelle 1:50 000 (Maroc nord occidental). Thèse D.E.S., Fac. Sc. Tétouan, $330 \mathrm{p}$.

COMÍN, F.A., M. MENÉNDEZ \& E. FORÉS -
1987- Salinidad y nutrientes en las lagunas costeras del delta del Ebro. Limnética, 3, Ass. Es. Limn., Spain, 1-8.

De LANGE, L. \& E. WESTINGA -1979- The distinction between Lemna gibba and Lemna minor on the basis of vegetative characters. Acta. Bot. Neerl., 28, 2-3, 169-176.

De La TORRE, J.R. -1955-El matorral en Yebala (Marruecos español). Estudio de las formaciones de matorral en la región de Yebala, su tratamiento y aprovechamiento en relación con la defensa y protección del suelo contra la erosion. Instituto de Estudios Africanos, CSIC, Madrid.

Den HARTOG, C. -1994- Suffocation of a littoral Zostera bed by Enteromorpha radianta. Aqu. Bot., 47, 21-28.

De THIOUX, M. -1989-Aménagement écologique des cours d'eau. Espèces aquatiques des eaux courantes, espèces des eaux, espèces ligneuses de la berge. Min. Rég. Wallonne (éd.), 3 Tomes.

EMBERGER, L. \& R. MAIRE -1941-Catalogue des plantes du Maroc (Spermatophytes et Ptéridophytes). Vol. IV, Imp. Minerva, Alger.

ENNABILI, A. \& M. ATER-1995-Phytoécologie et productivité de quelques macrophytes du marais Smir. in «Ater, M, \& M, Dakki (eds) : Les marais de Smir-Restinga. Ecologie et propositions d'aménagement ". Sous presse, Rabat.

EVANS, A.S., K.L. WEBB \& P.A. PENHALE 1986- Photosynthetic temperature aclimatation in two coexisting sea-grass, Zostera marina and Ruppia maritima L. Aquat. Bot., 24, 185-197.

FERNÁNDEZ-GONZÁLEZ, F., A. MOLINA y J. LOIDI - 1990- Los tarayales de la depresión del Ebro. Acta Bot. Malacitana, 15: 311-322.

FOUCART, T. -1985-Analyse factorielle. Programmation sur micro-ordinateur. Masson.

FRANÇOIS, J. - 1979- La jacinthe d'eau [Eichhornia crassipes (Mart.) Solms]. Fléau aquatique ou hydrophyte d'avenir. Ann. de Gembloux, 85, 73-81.

GONZÁLEZ GARCÍA, J.A. -1994-La flora marina de Melilla. Ensayos Melillensis, Melilla, 2, 212 pp.

GONZÁLEZ GARCÍA, J.A. \& F. CONDE POYALES -1995- Etudes comparatives sur le macrophytobenthos de trois lagunes côtières de la Méditerranée occidentale. Nova Hedwigia 
(Stuttgart), 61 (3-4) : 377-390.

GORENFLOT, R. -1986- Degrés et niveaux de variation du nombre chromosomique chez Phragmites australis (Cav.) Trin. ex Steud. Veröff. Geobot. Inst. ETH, Stiftung Rübel, Zürich, 87: 53-65.

GORENFLOT, R., J.M. HUBAC \& M. JAY -1984Le complexe polyplö̈de du Phragmites australis (Cav.) ex Steud. dans la région méditerranéenne. Webbia, 38: 715-721.

GOSSELINK, J-G. \& R.E. TURNER -1978- The role of hydrology in freshwater wetland ecosystems. in "R-E. Good, D-F. Whigham \& R-L. Simpson (eds.) : Freshwater Wetlands", Academic Press, New York, 63-78.

HASLAM, S.M. -1978-River plants. Cambridge Univ. Press.

HROUDOVÁ, S.H. \& P. ZÁKRAVSKY -1988Littoral vegetation of the Rozmberk fishpond. in "Z. Hroudová (ed.): Littoral Vegetation of the Rozmberk fishpond and its Mineral Nutrient Economy", Studie CSAV, Academia, Praha, 9, 23-60.

IONESCO, T. \& E. STEFANESCO -1967- La cartographie de la végétation de la région de Tanger. Al Awamia, 22 : 17-147, Rabat.

KETCHUM, B.H. -1954-Relation between circulation and planktonic populations in estuaries. Ecology, 35: 191-200.

KRAUSE,W. \& J.J. KING -1994- The ecological status of Lough Corrib, Ireland, as indicated by physiographic factors, water chemistry and macrophytic flora. Vegetatio, 110: 149-161.

LACHAVANNE, J.B. -1982- Influence de l'eutrophisation des eaux sur les macrophytes des lacs suisses: Résultats préliminaires. in "JJ. Symoens, S-S. Hooper \& P. Compère (eds.) : Studies on Aquatic Vascular Plants", Roy. Bot. Soc. Belgium, 333-339.

LANDOLT, E. -1975-Morphological differentiation and geographical distribution of the Lemna gibba-Lemna minor group. Aquat. Bot., 1: 345 363.

MAIRE, R. -1952/1980-Flore de l'Afrique du nord. Lechevalier, Paris.

MANDI, L. -1994- Marrakech wastewater purification experiment using vascular aquatic plants. Eichornia crassipes and Lemna gibba. Wat. Sc. Tech., vol. 29, 4: 283-287.

MANDI, L., J. DARLEY, J. BARBE \& BALEUX -
1992- Essai d'épuration des eaux usées de Marrakech par la jacinthe d'eau (charge organique, bactérienne et parasitologique). Rev. des Sciences de l'Eau, 5: 313-333.

MANDI, L., N. OUAZZANI, K. BOUHOUM \& A. BOUSSAID -1993- Wastewater treatemnt by stabilisation ponds with and without macrophytes under aride climate. Wat. Sc. Tech., vol. 28, 10: 177-181.

MENENDEZ, M. \& F.A. COMÍN -1989-Seasonal patterns of biomass variation of Ruppia cirrhosa (Petagna) Grande and Potamogeton pectinatus L. in a costal lagoon. Topics in Marine Biology, J.D. Ros (ed.), Scient. Mar., 53 (2-3),: 633-638.

MERIAUX, J-L. -1982- Espèces rares ou menacées des biotopes lacustres et fluviatiles du Nord de la France. in "J-J. Symoens, S-S. Hooper \& P. Compère (eds.) : Studies on Aquatic Vascular Plants”, Roy. Bot. Soc. Belgium, 398-402.

NAOMI, R. \& G.G. GANF - 1994- The influence of water regime on the performance of aquatique plants. Wat. Sc. Tech., vol. 29, 4, IAWQ, 127132.

NÈGRE, R. -1956- Note sur la végétation de quelques daya des Jbilete orientaux et occidentaux. Bull. Soc. Sc. Nat., Maroc, XXXVI: 229-241.

O.N.E.P. -1988-Contrôle de la pollution des eaux. Actions menées par l'ONEP. Rapport, Office Nationale de l'Eau Potable, Maroc.

PÉREZ-RAYA, F. \& J.M. LÓPEZ-NIETO -1991Vegetación acuática y helofítica de la depresión de Padul (Granada). Acta Bot. Malacitana, 16 (2): 373-389.

QUÉZEL, P. \& S. SANTA -1962/1963- Nouvelle flore de l'Algérie et des régions désertiques méridionales. CNRS, Paris.

RADOUX, M., D. CADELLI \& M. NEMKOVA 1995- L'optimisation des technologies extensives d'épuration des eaux usées sous climat tempéré (France) et sous climat sahélien (Sénégal). Word-wide Symposium : Pollution in large cities, science and technology for planing environmental quality, Venice/Padova, Italy, $11 \mathrm{p}$.

RADOUX, M. \& D. KEMP -1988- Epuration comparée des eaux usées domestiques par trois plantations hélophytiques et par un lagunage à microphytes sous un même climat tempéré. Acta Ecol. App., 9, 1, 25-38. 
REJMANKOVA, E. -1975- Comparaison of Lemna gibba and Lemna minor from the production ecological viewpoint. Aquat. Bot., 1, 423-427. RIVAS-MARTÍNEZ, S., M. COSTA, S. CASTROVIEJO y E. VALDÉS -1980Vegetación de Doñana (Huelva-España). Lazaroa, vol. 2, 5189.

SAUVAGE, Ch. -1971- Présentation botanique du Maroc. Al Awamia, 40-41: 29-214, Rabat.

SHALTOUT, K.H., H.F EL-KADY \& Y.M. ALSODANY -1995-Vegetation analysis of theMediterranean region of Nile Delta. Vegetatio, 116: 73-83.

STEVENSON, J.C. -1988- Comparative ecology of submersed grass beds in freshwater, estuarine and environments. Limnol. Oceanogr. 33 (4 part 2): 867-893.

STITOU, J. \& A. PULIDO-BOSCH -1995- Impacts anthropiques sur l'aquifère alluvial de Smir (Tétouan, Maroc). Hydrogéologie, 3 : 39-46.

TAHIRI, H. -1987-Quelques aspects de la méiose, de l'anatomie et de la résistance à la sécheresse dans le complexe polyploïde marocain $d u$ Phragmites australis (Cav.) Trin. ex Steud. Thèse D.E.S., Fac. Sc. Rabat, 152 pp.

TUTIN, T.G., N.A. BURGES, A.O. CHATER, J.R. EDMONDSON, G.H. HEYWOOD, D.M. MOORE, D.H. VALENTINE, S.M. WAITERS \& D.A. WEBB -1990/1993- Flora europaea. Cambridge University Press, UK.

VAN WIJK, R.J. -1988- Ecological studies on Potamogeton pectinatus L. I. General characteristics, biomass, production and life cycles under field conditions. Aquat. Bot., 31,: 211-258.

VANDEN BERGHEN, C. -1972- L'association à Schoenus nigricans et Aphyllanthes monspeliensis dans le département de l'Ardèche (France). Bul. Soc. Roy. Bot. Belgique, 105: 9-22.
VERHOVEN, J.T.A. -1980- The ecology of Ruppiadominated communities in Western Europe, II. Structure and dynamics of the macroflora and macrofauna communities. Aquat. Bot., 8: 1-85.

VUILLOT, M. \& C. BOUTIN -1987- Les systèmes rustiques d'épuration: aspects de l'expérience française; possibilités d'application aux pays en voie de développement. Trib. Cebedeau, 518 , 40, 21-31.

ZRID, R. -1988- Aspects des variabilités cytogénétique, palynologique et minérale de Phrcgmites australis (Cav.) Trin. ex Steud, en relation avec son évolution et son adaptation au Maroc. Thèse D.E.S., Fac. Sc. Rabat, 127 pp.

Aceptado para su publicación en Junio de 1996

Adresse des auteurs. Groupe d'Ecologie et Biologie des Populations, Département de Biologie, Faculté des Sciences, B. P. 2062, 93002 Tétouan-Maroc. 